\title{
Non-reducible descriptions for conditional Kolmogorov complexity
}

\author{
Andrej Muchnik* Alexander Shen ${ }^{\dagger} \quad$ Mikhail Ustinov ${ }^{\ddagger}$ \\ Nikolai Vereshchagin ${ }^{\S} \quad$ Michael Vyugin $₫$
}

\begin{abstract}
Assume that a program $p$ on input $a$ outputs $b$. We are looking for a shorter program $q$ having the same property $(q(a)=b)$. In addition, we want $q$ to be simple conditional to $p$ (this means that the conditional Kolmogorov complexity $K(q \mid p)$ is negligible). In the present paper, we prove that sometimes there is no such program $q$, even in the case when the complexity of $p$ is much bigger than $K(b \mid a)$. We give three different constructions that use the game approach, probabilistic arguments and algebraic arguments, respectively.
\end{abstract}

\section{Definitions and statements}

Let $a$ and $b$ be binary strings. Consider programs $p$ such that $p(a)=b$ (the program $p$ on input $a$ outputs $b$ ). What is the minimal length of such a program? If the programming language is chosen appropriately, this length is close to $K(b \mid a)$, the conditional Kolmogorov complexity of $b$ given $a$. We will ignore additive terms of order $O(\log n)$ where $n$ is the maximum length of the strings involved. With this precision all the versions of Kolmogorov complexity (the plain one, the prefix one etc.) coincide. For the definition of Kolmogorov complexity $K(b)$ and $K(b \mid a)$ we refer to the textbook [2].

*Institute of New Technologies; e-mail: muchnik@lpcs.math.msu.su. The work was supported by RFBR grants 04-01-00427, 06-01-00122a.

†The work was supported by CNRS (LIF, Marseille, France; Poncelet laboratory, Moscow), STINT foundation, Uppsala university (Sweden), Royal Holloway College (UK), RFBR (grants 02-01-22001, 03-01-00475, 06-01-00122a) and Scientific schools supporting council (grant NSh-358.2003.1); e-mail: shen@mccme.ru, alexander.shen@lif.univ-mrs.fr.

${ }_{\ddagger}^{\ddagger}$ Moscow State University, e-mail: mihail@ustinov.mccme.ru. The work was supported in part by the RFBR grants 02-01-22001, 03-01-00475, 06-01-00122a, NSh-358.2003.1.

$\S$ Moscow State University, e-mail: ver@mccme.ru. The work was supported in part by the RFBR grants 02-01-22001, 03-01-00475, 06-01-00122a, NSh-358.2003.1.

IMoscow State University, e-mail: misha@vyugin.mccme.ru, misha@cs.rhul.ac.uk. The work was supported in part by the RFBR grants 02-01-22001, 03-01-00475, 06-01-00122a, NSh-358.2003.1. 
To avoid references to a specific programming language we will consider "descriptions" instead of programs. A string $p$ is called a conditional description of a string $b$ given $a$ if $K(b \mid a, p)$ is negligible. Here $K(b \mid a, p)$ stands for the conditional complexity of $b$ given the pair $\langle a, p\rangle$. We will specify what is considered as "negligible" in each case.

For given $a$ and $b$ consider all strings $p$ such that $K(b \mid a, p) \approx 0$. One can easily verify that the length of any such $p$ is at least $K(b \mid a)$. This bound is tight. Both assertions are true with $O(\log n)$ precision; the same precision is required in the equality $K(b \mid a, p) \approx 0$.

We say that a description $q$ is a simplification of a description $p$ if $K(q \mid p) \approx 0$ with logarithmic precision. The relation $K(q \mid p)<\varepsilon$ is not transitive for a fixed $\varepsilon$ : $K(q \mid p)<\varepsilon$ and $K(r \mid q)<\varepsilon$ imply only that $K(r \mid p)<2 \varepsilon+O(\log n)$. However, this relation resembles a pre-ordering on strings and we are interested in the structure of the set of all conditional descriptions (for given $a, b$ ) with respect to this "pre-ordering".

The string $b$ itself is a conditional description of $b$ given $a$. Muchnik [1] has shown that, among all descriptions of $b$ relative to $a$, there exists a description of minimal length $(\approx K(b \mid a))$ that is at the same time a simplification of $b$. We will prove that this is not true in the general case (for arbitrary description $p$ instead of $b$ ): for some $a, b$ there is a description $p$ of complexity much larger than $K(b \mid a)$ that has no simplifications of length close to $K(b \mid a)$.

The exact statement is as follows:

Theorem 1. There exists a function $\varepsilon=\varepsilon(k, n)$ of order $O(\log (k+n))$ such that for all $k, n$ there are strings $a, b, p$ of lengths $n, 2 n, k$, respectively, having the following properties:

(a) $K(b \mid a, p) \leqslant \varepsilon$ ("the string $p$ is a conditional description of $b$ given $a$ ");

(b) $K(p \mid a) \geqslant k-\varepsilon$ ( ". . that has complexity close to its length $k$ even with condition $a$ ");

(c) there is no string $q$ such that $K(q) \leqslant k-n-\varepsilon, K(q \mid p) \leqslant n-\varepsilon$ and $K(b \mid a, q) \leqslant n-\varepsilon$ ( " $p$ has no simplifications of complexity $k-n$ ").

To be specific, in this theorem we have (quite arbitrary) chosen some relation between lengths of strings $a$ and $b$. The statement is interesting when $k \gg 2 n$ (e.g., if $k=4 n$ ); it says that we have a description $p$ that has high complexity $k$ (even if $a$ is known, so $p$ is a "random" string independent of $a$ ), but there is no simplification of $p$ that has complexity less than $k-n$ even if $k-n$ is much larger than the lower bound $K(b \mid a)$ which does not exceed $2 n$ (the length of $b$ ). Note that the word "negligible" is understood in a rather strict way when we guarantee it in (a), but quite liberal for the adversary in (c) (the bounds for $K(q \mid p)$ and $K(b \mid a, q)$ are $n-\varepsilon$ which is much more than $O(\log n)$.

In the preliminary version of this paper [3] we gave a more natural (but weaker) version of this statement and three different proofs of it (using games, probabilistic arguments and explicit algebraic construction). The current version includes improvements made by two of us (M.U. and A.M.); to avoid repetitions now we give the game proof of Theorem 1 (Section 2), a probabilistic proof of a stronger statement (Theorem 2 below) and a combinatorial proof of a constructive version of Theorem 1 (Theorem 3 below).

Before formulating these improved statements, let us note that Theorem 1 is only interesting if $k$ is bigger than $2 n$. If $k$ is close to $2 n$ (or is less) then the statement of Theorem 1 
becomes trivial. Indeed, if $k \leqslant 2 n$, let $a$ be the empty string, $p$ a random string of length $k$ (that is, $K(p) \geqslant k$ ) and $b=p 00 \ldots$. If some $q$ satisfies (c), then we get

$$
K(b) \leqslant K(q)+K(b \mid a, q)+O(\log n) \leqslant k-2 \varepsilon+O(\log n) .
$$

If $\varepsilon$ is big enough, this inequality contradicts the choice of $b$ having complexity $k+O(\log n) \leqslant$ $2 n+O(\log n)$. The same argument applies if $k$ is slightly larger than $n$, that is, $k=$ $2 n+O(\log n)($ in this case to construct $b$ we trim $p$ to the length $n$ ).

Theorem 1 asserts only that there exists a pair of strings $\langle a, b\rangle$ having a non-reducible description of $b$ given $a$. Surprisingly, it turns out that for all $\langle a, b\rangle$ and $k$, except for trivial cases, there exists a non-reducible description $p$ of $b$ relative to $a$ of complexity $k$. Here are the trivial cases:

(1) $K(a) \approx 0$; in this case the string $b$ is a simplification of every description of $b$ given $a$.

(2) $K(b \mid a) \approx 0$; in this case the empty string is a simplification of every description.

(3) $k$ is much less than $K(b \mid a)$; in this case there is no conditional description $p$ of $b$ relative to $a$ of complexity about $k$.

The exact statement is as follows:

Theorem 2. For all $k, n$ and all strings $a, b$ such that

$$
K(a)>n+\varepsilon, \quad K(b \mid a)>2 n+\varepsilon, \quad k>K(b \mid a)+\varepsilon
$$

there exists a string $p$ of length $k$ having the properties (a), (b) and (c) from Theorem 1. Here $\varepsilon$ is a function of $k$ and $K(a)$ of order $O(\log (k+K(a)))$.

Note that Theorem 2 implies Theorem 1. Indeed, let $\varepsilon$ in Theorem 1 be three times bigger than provided by Theorem 2 . Assume that $n, k$ are given. If $k<2 n+2 \varepsilon$ (where $\varepsilon$ is the function from Theorem 2) then $a, b$ can be constructed as in the remark above, without using Theorem 2. Otherwise let $n^{\prime}=n-2 \varepsilon$ and let $a$ and $b$ be independent random strings of lengths $n$ and $2 n$, respectively. Then $a, b, k, n^{\prime}$ satisfy the conditions of Theorem 2 and the string $p$ given by Theorem 2 together with $a, b$ satisfies Theorem 1 (with $3 \varepsilon$ in place of $\varepsilon)$.

The proof of Theorem 2 is given in Section 3; it uses probabilistic arguments. Finally, in Section 4 we give a combinatorial proof of the following "constructive" version of Theorem 1.

Theorem 3. Consider a finite field $F$ of cardinality $2^{n}$, a point $a \in F$ and a linear function $p(x)=p_{1} x+p_{2}$ from $F$ to $F$ such that $K\left(\left\langle p_{1}, p_{2}, a\right\rangle\right) \geqslant 3 n$. Let $b=p(a)$. Then $p=\left\langle p_{1}, p_{2}\right\rangle$ is a description of $b$ relative to a of complexity about $2 n$ that cannot be simplified: for all $i \leqslant n / 4$ there is no string $q$ such that $K(q) \leqslant 2 n-3 i-\varepsilon, K(q \mid p) \leqslant i-\varepsilon$ and $K(b \mid a, q) \leqslant i-\varepsilon$. Here $\varepsilon=O(\log n)$.

This theorem shows that for $3 i$-decrease in the complexity of the description we have to pay $i$ bits in the complexity of $K(q \mid p)$ or $K(b \mid a, q)$, so no significant simplification is possible if these complexities remain negligible.

The authors are grateful to all participants of Kolmogorov seminar of the Department of Mathematics (Mathematical Logic and Theory of Algorithms Division) at Moscow University. 


\section{Game approach}

Consider the following game we play against an adversary.

Let $P, Q, A$ and $B$ be finite sets (as we see later, they correspond to strings $p, q, a, b$ respectively). On our moves we construct a partial function $\Xi: P \times A \rightarrow B$. At the start of the game the function $\Xi$ is empty, and on each move we may define the value of $\Xi$ at one point (once defined values cannot be changed later). Or we may skip the move, that is, we may leave $\Xi$ unchanged.

The adversary on his moves constructs multi-valued functions $\Phi: P \rightarrow Q$ and $\Psi: Q \times A \rightarrow$ $B$. That is, the values of $\Phi$ are subsets of $Q$, and the values of $\Psi$ are subsets of $B$. Initially $\Phi$ and $\Psi$ are empty (all their values are empty). At each move the adversary may add one new value to $\Phi$ (adding a new element to $\Phi(p)$ for some $p$ ) or $\Psi$ (adding a new element to $\Psi(q, a)$ for some $q, a)$. The existing elements cannot be removed. The adversary also may skip the move.

The adversary must obey the following rules: the function $\Phi$ takes on every argument at most $\varphi$ values (i.e., $\# \Phi(p) \leqslant \varphi$ for any $p \in P$ ) and the function $\Psi$ takes on every argument at most $\psi$ values $(\# \Psi(q, a) \leqslant \psi$ for any $q, a)$.

Players' moves alternate. Obviously, each player can make only finite number of nontrivial moves (moves that change the functions). Thus after a certain move all the three functions remain unchanged. The result of the game is defined as follows: we win if there exist $p \in P, a \in A$ and $b \in B$ such that $\Xi(p, a)=b$ and $p, a, b$ are not "covered" by the adversary: the latter means that there is no $q \in \Phi(p)$ such that $b \in \Psi(q, a)$.

So the game is determined by the sets $A, B, P$ and $Q$ (actually, only their cardinalities matter) and the parameters $\varphi$ and $\psi$. We represent the function $\Xi$ as a table with $\# P$ rows and $\# A$ columns. The cells of this table initially are empty; we fill them by elements of $B$ (each cell may contain at most one element).

The adversary fills the table for function $\Psi$. It has $\# Q$ rows of the same length $\# A$ as in our table. Each cell may contain up to $\psi$ elements of $B$. The adversary also constructs the function $\Phi$. It is convenient to represent this function by arrows going from row $p$ of our table to all rows of adversary's table that belong to $\Phi(p)$. The out-degree is bounded by $\varphi$. We win if our table has a non-covered cell. A cell $\langle p, a\rangle$ is covered if its row is connected by an arrow to a row of adversary's table that has in the same column the same element of $B$ (and, may be, some other elements). See Fig. 1.

The proof is based on the following simple observation:

Lemma 1. If $\varphi \cdot \psi<\# B$ and $\varphi \cdot \# P+\psi \cdot \# A \cdot \# Q<\# A \cdot \# P$ then we have a winning strategy in the game.

Proof. The first inequality guarantees that if $\Xi$ is not yet defined on a pair $\langle p, a\rangle$ then we can choose a value $b=\Xi(p, a)$ so that the cell $\langle p, a\rangle$ is not covered (at the current step). Indeed, for each of at most $\varphi$ values $q \in \Phi(a)$ there exist at most $\psi$ values $b \in \Psi(q, a)$, so there exists $b$ that is different from all those values.

Choosing $b$ in this way (assuming that there are empty slots in $\Xi$-table), we guarantee that after each our move there exists a non-covered cell $\langle p, a\rangle$. Our move is non-trivial only 


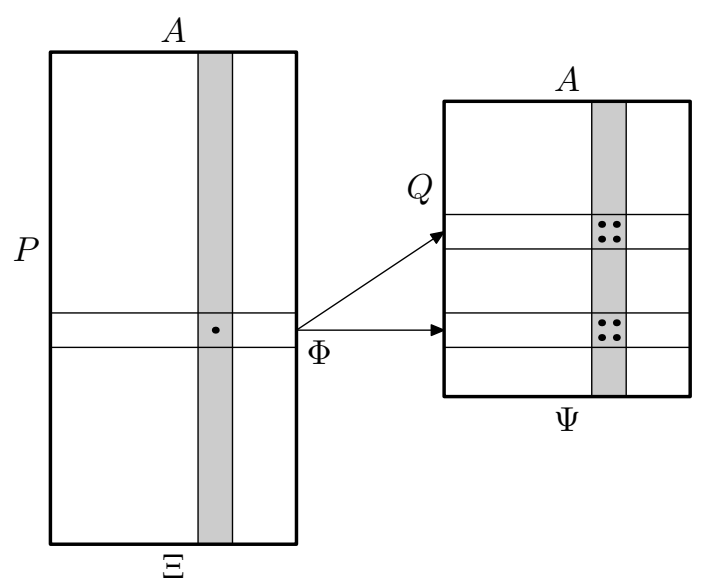

Figure 1: Cells of our table $\Xi$ and adversary's table $\Psi$ are filled with elements of $B$; each row of $\Xi$ has at most $\varphi$ outgoing edges, each cell of $\Psi$ contains at most $\psi$ elements.

when the previous adversary's move is non-trivial. The second inequality guarantees that the number of cells in $\Xi$-table is greater than the number of adversary's non-trivial moves (so the empty slots do exist). Indeed, for each of $\# P$ arguments the value of $\Phi$ may be changed at most $\varphi$ times and for each of $\# A \cdot \# Q$ pairs $\langle q, a\rangle$ the value of $\Psi$ may be changed at most $\psi$ times.

Hence after every adversary's non-trivial move we can find an empty cell in $\Xi$-table and enter a value in it so that the cell becomes non-covered. The lemma is proved.

Now we prove Theorem 1 using Lemma 1 . Let $A, B$ be the set of all strings of lengths $n, 2 n$, respectively. Let $P$ be the set of all strings of length $k$ and let $Q$ be the set of all strings of length less than $k-n$.

Let us fix the adversary's strategy now. Assume that the adversary includes in $\Phi(p)$ (one by one) all $q \in Q$ such that $K(q \mid p)<n-2$, and includes in $\Psi(q, a)$ all the strings $b \in B$ such that $K(b \mid a, q)<n-2$. One can do this effectively given $n$, as the function $K$ is upper semi-computable (that is, the set $\{\langle x, y, l\rangle \mid K(x \mid y)<l\}$ is recursively enumerable). This strategy does not violate the rules provided $\varphi=\psi=2^{n-2}$.

Let us verify that the conditions of Lemma 1 are satisfied:

$$
\varphi \cdot \psi=2^{2 n-4}<2^{2 n}=\# B
$$

and

$$
\varphi \cdot \# P+\psi \cdot \# A \cdot \# Q=2^{n-2+k}+2^{(n-2)+n+(k-n)}=2^{n-1+k}<\# A \cdot \# P .
$$

Therefore, by Lemma 1, we have a winning strategy in the game.

The winning strategy is computable given $n, k$. Applying it against the adversary's strategy described above we obtain a function $\Xi$ that is computable given $n, k$ (as the adversary's moves are computable, so are ours). To be precise we should write $\Xi_{n k}$ indicating the dependence on $n, k$; complexity of algorithm that computes $\Xi_{n k}$ is $O(\log (n+k))$ since $\Xi_{n k}$ is 
determined by $n, k$. Since our strategy is a winning one, there exists a cell $\langle p, a\rangle$ that is not covered after all non-trivial moves are performed. (It depends on $n, k$ in a non-computable way, as we do not know which of the adversary's moves is the last non-trivial one.)

Let $b=\Xi(p, a)$ be the element in the "winning" cell of our table. Then $K(b \mid a, p)=$ $O(\log (n+k))$. As the cell $(p, a)$ is not covered, there is no string $q$ of length less than $k-n$ such that $K(q \mid p)<n-2$ and $K(b \mid a, q)<n-2$. This is weaker than required: we want the statement to be true for all $q$ of complexity less than $k-n-\varepsilon$ (and not the length less than $k-n)$. However it is easy to fix this. Replacing $q$ by its shortest description we increase $K(b \mid a, q)$ and $K(q \mid p)$ by $O(\log (n+k))$ and this increase can be compensated by the choice of $\varepsilon$. It remains to fix only one problem: we want the complexity of $p$ given $a$ to be at least $k-\varepsilon$ and the rules of the game do not provide any guarantee for this.

Let us change the game allowing the adversary one more type of moves: at any step adversary may remove (= "mark as unusable") any cell of the table of $\Xi$, but in every column the total number of removed cells should not exceed \#P/2 (so at least half of cells remain intact). In the winning rule we require that the cell $\langle p, a\rangle$ has not been removed by the adversary. For the modified game the statement of Lemma 1 is changed as follows: in the right hand side of the inequality $\varphi \cdot \# P+\psi \cdot \# A \cdot \# Q<\# A \cdot \# P$ the term $\# A \cdot \# P$ is replaced by $\# A \cdot \# P / 2$. The modified lemma is still true: Indeed, if we cannot perform any move then we have used all the non-removed cells, thus we have done $\# A \cdot \# P / 2$ moves. And the conditions of the modified lemma are still fulfilled.

Other changes are as follows: the adversary removes all the cells $\langle p, a\rangle$ such that $K(p \mid a)<$ $k-1$. It is clear that, in every column, at most half cells are removed. After this modification we know that for the winning cell $\langle p, a\rangle$ the complexity of $p$ given $a$ is at least $k-1$, and the theorem is proved.

\section{Probabilistic approach}

The strategy used in the previous section is quite simple: after looking at the adversary's move we check whether all uncovered cells have disappeared; if yes, we create a new uncovered cell. In fact, a completely different "blind" strategy could be also successful: we can decide in advance what should be in our table and completely ignore the moves of the adversary.

To implement this approach we need to prove that there exists a function $\Xi$ such that for any adversary moves there exists an uncovered cell. This can be done by a probabilistic argument: for each adversary behavior we prove that only a tiny fraction of functions $\Xi$ do not work, so the probability that a randomly chosen function does not work for a given adversary behavior is very small and the sum of these probabilities over all possible behaviors is less than 1.

Theorem 1 can be proved in this way, but to avoid repetitions we provide the proof for the stronger Theorem 2. (A weaker version of Theorem 2 can also be proved with a game argument, see [4].) Since we want to prove that for all pairs $\langle a, b\rangle$ (under some conditions) there exists a program that cannot be simplified, we now consider pairs $\langle a, b\rangle$ instead of cells $\langle p, a\rangle$. 
Assume that finite sets $A, B, P, Q$ are fixed. (They will play the same role as before.) Consider functions $\Xi: P \times A \rightarrow B$ and multi-valued functions $\Phi: P \rightarrow Q, \Theta: A \rightarrow P$ and $\Psi: Q \times A \rightarrow B$ having at most $\varphi, \theta$ and $\psi$ values, respectively, for each argument. (The functions $\Phi$ and $\Psi$ play the same role as before. The value $\Theta(a)$ corresponds to the list of removed cells in column a.)

Call a pair $\langle a, b\rangle \in A \times B$ covered (for given $\Xi, \Phi, \Theta, \Psi)$ if for all $p \in P \backslash \Theta(a)$ such that $\Xi(p, a)=b$ there exists $q \in \Phi(p)$ such that $b \in \Psi(q, a)$. Let $B(a)$ denote the set of all $b \in B$ such that the pair $\langle a, b\rangle$ is covered.

Fix two natural parameters $\alpha \ll \# A$ and $\beta \ll \# B$ and call a function $\Xi$ a winning function (cf. the game described above) if for all multi-valued $\Phi, \Theta$ and $\Psi$ satisfying given bounds on the number of values, the inequality

$$
\#\{a \in A \mid \# B(a) \geqslant \beta\}<\alpha
$$

holds. If a function $\Xi$ is winning, we can put its values in the table ignoring the adversary's moves and guarantee that we win and, moreover, that for almost all $a \in A$ (with less than $\alpha$ exceptions) for almost all $b \in B$ (with less than $\beta$ exceptions) the pair $\langle a, b\rangle$ is not covered.

The following lemma says that (under some restrictions on the parameters) the winning function exists. Its proof uses probabilistic arguments.

Lemma 2. Let $s=(\beta-\varphi \psi) / \# B$. If

$$
2^{\# A}\left(2^{\# B}(1-s)^{\# P-\theta}\right)^{\alpha} \cdot(\# Q)^{\varphi \cdot \# P} \cdot(\# P)^{\theta \cdot \# A} \cdot(\# B)^{\psi \cdot \# Q \cdot \# A}<1
$$

then there is a winning function.

Let us first prove Theorem 2 using this lemma; then we give a proof for Lemma 2 and therefore complete the proof of Theorem 2 .

Given $a, b, n, k$ satisfying the conditions of the theorem (for $\varepsilon$ to be chosen later), let $A, B$ be the sets of strings of length $K(a), K(b \mid a)$ respectively. Let $\delta$ be a function of $K(a), k$ of order $O(\log (K(a)+k))$ to be chosen later. Let $P$ be the set of strings of length $k$ and $Q$ the set of strings of length less than $k-n-3 \delta$. Fix the functions $\Phi, \Theta, \Psi$ as follows:

$$
\Phi(p)=\{q \mid K(q \mid p)<n\}, \Theta\left(a^{\prime}\right)=\left\{p \mid K\left(p \mid a^{\prime}\right)<k-3 \delta\right\}, \Psi\left(q, a^{\prime}\right)=\left\{b \mid K\left(b \mid q, a^{\prime}\right)<n\right\} .
$$

They satisfy the requirements for the number of values provided $\varphi=\psi=2^{n}$ and $\theta=$ $2^{k-3 \delta}$. Finally, let $\alpha=2^{K(a)-\delta}, \beta=2^{K(b \mid a)-\delta}$, so the "exceptional" values form a $2^{-\delta}$-fraction among all $a$ (or $b)$.

First we verify that if $\varepsilon$ is much greater than $\delta$ then the chosen parameters satisfy the conditions of Lemma 2. (Later we show that the conclusion of Lemma 2 implies the statement of Theorem 2.)

As $K(b \mid a)>2 n+\varepsilon \gg 2 n+\delta$, we have $s=\left(2^{K(b \mid a)-\delta}-2^{2 n}\right) 2^{-K(b \mid a)}>2^{-\delta-1}$. Therefore,

$$
1-s \leqslant 1-2^{-\delta-1} \leqslant e^{-2^{-\delta-1}}<2^{-2^{-\delta-1}} \text {. }
$$


As $\# P=2^{k} \gg 2^{k-3 \delta}=\theta$, we have $\# P-\theta>\# P / 2$, thus, it is enough to show that

$$
2^{\# A}\left(2^{\# B} 2^{-2^{-\delta-1} \cdot \# P / 2}\right)^{\alpha} \cdot(\# Q)^{\varphi \cdot \# P} \cdot(\# P)^{\theta \cdot \# A} \cdot(\# B)^{\psi \cdot \# Q \cdot \# A}<1
$$

Let us focus on the exponents in this inequality. The inequality is true if all the positive exponents are much less than the negative exponent, which is equal to $2^{-\delta-1} \cdot \# P \cdot \alpha / 2=$ $2^{-\delta-2+k+(K(a)-\delta)}$. So we need to show that

$$
\begin{aligned}
\# A=2^{K(a)} & \ll 2^{-2 \delta-2+k+K(a)}, \\
\# B \cdot \alpha=2^{K(b \mid a)+K(a)-\delta} & \ll 2^{-2 \delta-2+k+K(a)}, \\
\varphi \cdot \# P=2^{n+k} & \ll 2^{-2 \delta-2+k+K(a)}, \\
\theta \cdot \# A=2^{k-3 \delta+K(a)} & \ll 2^{-2 \delta-2+k+K(a)}, \\
\psi \cdot \# Q \cdot \# A=2^{n+(k-n-3 \delta)+K(a)} & \ll 2^{-2 \delta-2+k+K(a)} .
\end{aligned}
$$

The first condition is true, as $k>\varepsilon \gg \delta$. The second one is true, as $k>K(b \mid a)+\varepsilon$. The third one is true, as $K(a) \geqslant n+\varepsilon$. The remaining two inequalities are obviously true provided $\delta$ is large enough. Note that the difference between the negative exponent and all the positive ones is at least $\delta-O(1)$. If $\delta=O(\log (k+K(a))$ is chosen appropriately the difference is large enough to compensate the difference in bases: $2^{\delta} \gg \log \# Q, \log \# P, \log \# B$.

By Lemma 2 there is a winning function. To complete the argument, we need not just a winning function, but a winning function $\Xi$ of Kolmogorov complexity of order $O(\log (K(a)+$ $k)$ ), that is, the Kolmogorov complexity $K(\Xi)$ of the graph of $\Xi$ should be $O(\log (K(a)+k))$. To prove that there is a winning function $\Xi$ such that $K(\Xi)=O(\log (K(a)+k))$ we can use the following (very general) argument: By a very long (but finite) exhaustive search we can check whether a given function is winning or not (checking all $\Theta, \Phi$ and $\Psi$ ). Thus we can probe all the functions $\Xi$ in some natural order until we find the first winning one. To run this algorithm we need only to know $n, k, K(a), K(b \mid a)$. As all these numbers are less than $K(a)+k$, the first winning function has Kolmogorov complexity $O(\log (K(a)+k))$.

As $\Xi$ beats the chosen $\Phi, \Theta, \Psi$ we have

$$
\#\left\{a^{\prime} \in A \mid \# B\left(a^{\prime}\right) \geqslant 2^{K(b \mid a)-\delta}\right\}<2^{K(a)-\delta} .
$$

Let us see what means that a pair $\left\langle a^{\prime}, b^{\prime}\right\rangle$ is not covered for chosen $\Phi, \Theta, \Psi$. This means that

there exists $p$ of length $k$ and complexity at least $k-3 \delta$ such that $\Xi\left(p, a^{\prime}\right)=b^{\prime}$ and there is no $q$ of length less than $k-n-3 \delta$ such that $K(q \mid p)<n$ and $K\left(b \mid q, a^{\prime}\right)<n$.

Note that $b^{\prime}=\Xi\left(p, a^{\prime}\right)$ implies $K\left(b^{\prime} \mid p, a^{\prime}\right)=O(\log (K(a)+k))$.

Now we consider any pair $\langle a, b\rangle$ that satisfies the assumption of Theorem 2 . We show that the pair 〈the shortest program for $a$, the shortest program for $b$ given $a\rangle$ is not covered (i.e., has the property printed in italics above).

Indeed, covered pairs can be enumerated given $k, n, K(a), K(b \mid a)$. This implies that all $a^{\prime}$ with $\# B\left(a^{\prime}\right)>2^{K(a)-\delta}$ can be enumerated too. As $\Xi$ is winning, the number of such $a^{\prime}$ is 
less than $2^{K(a)-\delta}$ hence every such $a^{\prime}$ has complexity at most $K(a)-\delta+O(\log (K(a)+k))<$ $K(a)-\delta / 2$, provided $\delta$ is large enough. For remaining $a^{\prime}$ we have $B\left(a^{\prime}\right)<2^{K(b \mid a)-\delta}$, hence for all $b^{\prime} \in B\left(a^{\prime}\right)$ it holds

$$
K\left(b^{\prime} \mid a^{\prime}\right)<K(b \mid a)-\delta+O(\log (K(a)+k))<K(b \mid a)-\delta / 2 .
$$

So for any covered pair at least one of these two inequalities is true.

On the other hand, if $a^{\prime}$ is the shortest program for $a$ and $b^{\prime}$ is the shortest program for $b$ given $a$ and $\delta$ is large enough then $K\left(a^{\prime}\right)>K(a)-\delta / 2$ and $K\left(b^{\prime} \mid a^{\prime}\right)>K(b \mid a)-\delta / 2$ hence the pair $\left\langle a^{\prime}, b^{\prime}\right\rangle$ is not covered. That is, the statement above (printed in italics) is true for $\left\langle a^{\prime}, b^{\prime}\right\rangle$. If we replace now $a^{\prime}, b^{\prime}$ by $a, b$, respectively, we change all the complexities involved by at most $O(\log (K(a)+k))$. In this way we obtain almost the statement in the conclusion of Theorem 2.

The only problem left is that in the quoted statement $q$ ranges over strings of length less than $n-k-3 \delta$ and not of complexity less than $n-k-\varepsilon$, as in Theorem 2. This is fixed in the same way as in the proof of Theorem 1: changing $q$ to its shortest program increases $K(b \mid a, q)$ and $K(q \mid p)$ by $O(\log (K(a)+k))$.

It remains to prove Lemma 2.

Proof of Lemma 2. It is clear that without loss of generality we may assume that the functions $\Phi, \Theta$ and $\Psi$ always take maximum allowed number of values (if $\Xi$ wins in this case, it wins always).

First fix some $\Phi, \Theta$ and $\Psi$ and prove that the probability that a randomly chosen function $\Xi$ does not beat $\Phi, \Theta$ and $\Psi$ is less than the first factor $2^{\# A}\left(2^{\# B}(1-s)^{\# P-\theta}\right)^{\alpha}$ in the inequality (assuming that all functions $\Xi$ are equiprobable).

To this end we give an upper bound for the probability that $\# B(a) \geqslant \beta$ for a fixed $a$. If $\# B(a) \geqslant \beta$ then there is a set $B^{\prime} \subset B$ of cardinality $\beta$ such that all pairs in $\{a\} \times B^{\prime}$ are covered. Fix any such $B^{\prime}$; let us provide an upper bound for the probability that all pairs in $\{a\} \times B^{\prime}$ are covered. If this happens, then for all $p \in P \backslash \Theta(a)$, the value $\Xi(p, a)$ gets outside $B^{\prime}$ or gets into the set $\cup_{q \in \Phi(a)} \Psi(q, a)$. For fixed $p \in P$ the probability of this event is at most $\left(1-\# B^{\prime} / \# B\right)+\varphi \psi / \# B=1-s$, as $\Xi(p, a)$ is chosen at random in $B$ and the number of elements in $\cup_{q \in \Phi(a)} \Psi(q, a)$ is at most $\varphi \psi$. Since for different $p$ the values $\Xi(p, a)$ are independent, all pairs in $\{a\} \times B^{\prime}$ are covered with probability at most $(1-s)^{\# P-\Theta(a)} \leqslant(1-s)^{\# P-\theta}$. By the union bound the probability that $\# B(a) \geqslant \alpha$ is at most $2^{\# B}(1-s)^{\# P-\theta}$, since the number of different $B^{\prime}$ does not exceed $2^{\# B}$.

Again, by union bound the probability that the number of $a$ with $\# B(a) \geqslant \beta$ is at least $\alpha$ does not exceed (the number of $A^{\prime} \subset A$ of cardinality $\alpha$ ) times (the probability that $\# B(a) \geqslant \beta$ for all $\left.a \in A^{\prime}\right)$. The number of $A^{\prime}$ is less that $2^{\# A}$. The probability that $\# B(a) \geqslant \beta$ for all $a \in A^{\prime}$ is at most $\left(2^{\# B}(1-s)^{\# P-\theta}\right)^{\alpha}$, as the values $\Xi(p, a)$ are chosen independently for different $a$. Multiplying these two numbers we obtain the first factor $2^{\# A}\left(2^{\# B}(1-s)^{\# P-\theta}\right)^{\alpha}$ in the inequality.

Is is easy to see that the other three factors are the upper bounds for the number of different functions $\Phi, \Theta$ and $\Psi$, respectively. 
As it was mentioned above, Theorem 2 implies Theorem 1. Thus we obtain a new proof of Theorem 1. What is the advantage of this (more complicated) proof? It shows that Theorem 1 can be strengthened as follows: for every oracle $X$ there exist $p, a, b$ satisfying condition (a) of the theorem (unchanged, without the oracle) such that $K^{X}(p \mid a) \geqslant k-\varepsilon$ and there is no $q$ for which both $K^{X}(q \mid p)$ and $K^{X}(b \mid a, q)$ are less than $n-\varepsilon$. Indeed, our winning function beats any adversary's strategy and its construction (and the inequality $K(b \mid a, p) \approx 0)$ does not depend on the enemy's strategy. The same applies to Theorem 2: the items (b) and (c) in its conclusion can be relativized by any oracle $X$ provided its condition is relativized by the same oracle; item (a) remains unrelativized. [Instead of relativizing the Kolmogorov complexity by an oracle one can add any string as the extra condition in $K(p \mid a), K(q \mid p)$ and $K(b \mid a, q)$.]

\section{Algebraic construction}

In the previous section we have proved the existence of a winning function; moreover, the first winning function (in some ordering) has small complexity since it can be found by an exhaustive search. However, the proof does not tell us what properties make this function winning. So in this section we give a more explicit argument.

Unfortunately, we do not know a specific example of a winning function as defined in the previous section (in the proof of Theorem 2). So we have to return to the (slightly modified) framework of Theorem 1 where we provided a non-reducible descriptions only for some $a$ and $b$, not for (almost) all pairs $a, b$.

As in Section 2, let $\Xi: P \times A \rightarrow B$ be a function and let $\Phi: P \rightarrow Q$ and $\Psi: Q \times A \rightarrow B$ be multi-valued functions that have at most $\varphi$ and $\psi$ values, respectively. We say that a pair (cell) $\langle p, a\rangle$ is covered if there exists $q \in \Phi(p)$ such that $\Xi(p, a) \in \Psi(q, a)$. Fix some $\beta \in(0 ; 1)$ and call $\Xi$ winning if for all $\Phi, \Psi$ the fraction of covered cells is at most $\beta$, i.e., the number of covered cells is at most $\beta \cdot \# P \cdot \# A$.

We could use the probabilistic arguments to show that there exists a winning function (under certain conditions on $A, B, P, Q, \varphi, \psi, \beta$ ). Then we could find a winning function by an exhaustive search. But now we would like to have a more explicit example of a winning function.

The statement of Theorem 3 is based on the following example. Let $A=B=F, P=F^{2}$, where $F$ is a finite field. Then the function $\Xi\left(\left(p_{1}, p_{2}\right), a\right)=p_{1}+p_{2} a$ is a winning one (for some natural values of the parameters). This happens because of some combinatorial properties of that function.

Consider a function $\Xi: P \times A \rightarrow B$. For every $p \in P$ consider the corresponding line in the table $\Xi$, that is, the function $\Xi_{p}: A \rightarrow B$ defined as $\Xi_{p}(a)=\Xi(p, a)$. We require that the functions $\Xi_{p}$ for different $p$ (=different lines of the table $\Xi$ ) are far away from each other. This requirement seems natural: if there are many $a$ 's such that $\Xi_{p}(a)=\Xi_{r}(a)$, then the adversary may use the same $q$ for $p$ and $r$.

Formally speaking, we give the following

Definition. A function $\Xi$ is $\alpha$-regular if for all $p \neq r$ the number of $a \in A$ such that 
$\Xi_{p}(a)=\Xi_{r}(a)$ is at most $\alpha$ (=if the Hamming distance between corresponding lines is at least $\# A-\alpha)$.

Lemma 3. Every $\alpha$-regular function is winning provided

$$
4 \varphi \psi^{2} \cdot \# Q \leqslant \beta \# P, \quad 4 \varphi \psi \sqrt{\alpha} \leqslant \beta \sqrt{\# A}
$$

Proof. First we reduce the general case to the case $\psi=1$. To this end we replace every line in the table $\Psi$ by $\psi$ lines (that contain the same elements of $B$ as the old line, one element per cell). The height of the table, $\# Q$, becomes $\psi$ times bigger and the function $\Phi$ has now $\psi$ times more values (each arrow is replaced by $\psi$ arrows). So $\varphi$ is replaced by $\tilde{\varphi}=\varphi \psi$. More precisely, if a function $\Xi$ is winning for $\tilde{Q}=\{1, \ldots, \psi\} \times Q, \tilde{\varphi}=\varphi \psi$ and $\tilde{\psi}=1$ (all other parameters remain unchanged) then $\Xi$ is winning with the original parameters. Indeed, every $\Phi, \Psi$ for the original game can be transformed into $\tilde{\Phi}, \tilde{\Psi}$ for the modified game: let $\tilde{\Phi}(p)$ be the set $\{\langle i, q\rangle \mid q \in \Phi(p)\}$, and let $\tilde{\Psi}(\langle j, q\rangle, a)$ be equal to the $j$ th value of $\Psi(q, a)$, in some order. If $\Xi$ beats $\tilde{\Phi}, \tilde{\Psi}$ then it beats also $\Phi, \Psi$.

The conditions of the lemma translate into the inequalities

$$
4 \tilde{\varphi} \cdot \# \tilde{Q} \leqslant \beta \# P \text { and } 4 \tilde{\varphi} \sqrt{\alpha} \leqslant \beta \sqrt{\# A} .
$$

So we can assume that $\psi=1$ from now on and continue the proof of Lemma 3.

Let us split a $\varphi$-valued function $\Phi$ into $\varphi$ single-valued functions $\Phi_{1}, \ldots, \Phi_{\varphi}$. Each $\Phi_{i}$ covers some cells of the table $\Xi$, and it in enough to show that the fraction of elements covered by every $\Phi_{i}$ is less than $\beta / \varphi$.

Why any single-valued function $\Phi_{i}$ covers few cells? The reason is that $\# Q$ is much less than $\# P$, thus in many cases the same line of the table $\Psi$ must correspond to many lines of the table $\Xi$. By our assumption the lines of $\Xi$ have small intersection and hence cannot be easily covered by the same $\Psi$-line. The formal argument use the following simple bound:

Lemma 4. Assume that a family of $k$ subsets of an s-element set is given such that every two subsets in this family have at most $\alpha$ common elements. Then the sum of cardinalities of all the subsets in the family is at most

$$
2 s+2 k \sqrt{s \alpha} .
$$

Remark: For small $k$ the first term of the sum $2 s+2 k \sqrt{s \alpha}$, not depending on $k$, is the main term; for large $k$ the second term, linear in $k$, is the main term; two terms are equal for $k=\sqrt{s / \alpha}$.

Proof. Let $s_{1}, \ldots, s_{k}$ be the cardinalities of the given subsets. The inclusions-exclusions formula implies that

$$
s \geqslant s_{1}+s_{2}+\ldots+s_{k}-k^{2} \alpha
$$

(there are at most $k^{2}$ pairwise intersections, each of cardinality at most $\alpha$ ). Therefore

$$
s_{1}+\ldots+s_{k} \leqslant s+k^{2} \alpha .
$$


If $k \leqslant \sqrt{s / \alpha}$ then the second term $\left(k^{2} \alpha\right)$ is bounded by $s$ and the sum $s+k^{2} \alpha$ is at most $2 s$. Hence the inequality of the lemma is true for all $k \leqslant \sqrt{s / \alpha}$. For $k=\sqrt{s / \alpha}$ we have also $s_{1}+\ldots+s_{k} \leqslant 2 k \sqrt{s \alpha}$, as in this case $2 k \sqrt{s \alpha}=2 s$. Since the right hand side of the last inequality is linear in $k$, the inequality is true for all $k \geqslant \sqrt{s / \alpha}$. To demonstrate this let us delete from the sum $s_{1}+\ldots+s_{k}$ all terms except for the $\sqrt{s / \alpha}$ largest ones. As the average of remaining terms is not smaller than the average of all terms, we are done.

In fact this proof works only if $\sqrt{s / \alpha}$ is an integer. This is not really important since one can easily adapt the arguments below and use Lemma 2 only for integer case, but we can still prove Lemma 2 in general case using more careful bounds. Namely, $s_{1}+\ldots+s_{k} \leqslant$ $s+(k(k-1) / 2) \alpha$, since there are at most $k(k-1) / 2$ pairwise intersections. Then for $k=\lceil\sqrt{s / \alpha}\rceil$ we have

$$
s+(k(k-1) / 2) \alpha \leqslant s+\frac{1}{2} \sqrt{s / \alpha}(\sqrt{s / \alpha}+1) \alpha \leqslant s+\frac{1}{2} \sqrt{s}(\sqrt{s}+\sqrt{\alpha}) \leqslant 2 s \leqslant 2 k \sqrt{s \alpha}
$$

(we may assume without loss of generality that $\alpha \leqslant s$ ), and the proof can be completed as before.

Let us continue the proof of Lemma 3 . If $k$ different lines of $\Xi$ are mapped by $\Phi_{i}$ onto one line of $\Psi$, then the sets of covered columns in any two of these lines have at most $\alpha$ common elements. Hence the total number of covered cells in these $k$ lines is at most

$$
2 \# A+2 k \sqrt{\# A \alpha} \text {. }
$$

We have to sum this numbers for all $\# Q$ elements that can be values of the function $\Phi_{i}$, that is, over all lines of table $\Psi$.

The first terms sum up to $2 \# A \cdot \# Q$, the second ones sum up to $2 \cdot \# P \sqrt{\# A \cdot \alpha}$. So the total number of cells covered by each $\Phi_{i}$ is at most

$$
2 \# A \cdot \# Q+2 \cdot \# P \sqrt{\# A \alpha} .
$$

Recalling that there are $\varphi$ functions $\Phi_{i}$ we conclude that the number of covered cells is at most

$$
2 \varphi \cdot \# A \cdot \# Q+2 \varphi \cdot \# P \sqrt{\alpha \cdot \# A} .
$$

The conditions of the lemma imply that this is less than $\beta \cdot \# P \cdot \# A$.

Assume now that $n, i$ satisfy conditions of Theorem 3, i.e., $i \leqslant n / 4$. Let $A=B$ be the field of cardinality $2^{n}$, and let $P$ be the set of all linear functions $\left(x \mapsto p_{1} x+p_{2}\right)$ from $A$ to $A$. A linear function is determined by its two coefficients $p_{1}, p_{2}$, thus $\# P=2^{2 n}$. We let $\alpha=1$, as if two linear functions coincide in two points then they coincide everywhere.

Let $Q=\{0,1\}^{2 n-3 i}$ and $\varphi=\psi=2^{i-\gamma}, \beta=2^{-\gamma}$ where $\gamma=O(\log n)$ is to be specified later. Let us verify that the conditions of Lemma 3 are satisfied. We need to check that

$$
\begin{aligned}
4 \varphi \psi^{2} \cdot \# Q=2^{2+3(i-\gamma)+(2 n-3 i)}=2^{2+2 n-3 \gamma} & \ll 2^{2 n-\gamma}=\beta \cdot \# P, \\
4 \varphi \psi \cdot \sqrt{\alpha}=2^{2+2(i-\gamma)} & \ll 2^{n / 2-\gamma}=\beta \cdot \sqrt{\# A} .
\end{aligned}
$$


The first inequality is evident, the second one is guaranteed by the condition $i \leqslant n / 4$. Now consider the functions

$$
\Phi(p)=\{q \mid K(q \mid p)<i-\gamma\}, \quad \Psi(q, a)=\{b \mid K(b \mid q, a)<i-\gamma\} .
$$

These $\Phi, \Psi$ satisfy the requirements on the number of values. Therefore, by Lemma 3 the number of covered cells is at most $2^{3 n-\gamma}$. As covered cells can be enumerated given $n$, the complexity of every covered cell is at most $3 n-\gamma+O(\log n)<3 n$ provided $\gamma$ is large enough. Thus every random cell $\langle p, a\rangle$ is not covered. This means that there is no $q$ of length less than $2 n-3 i$ with $K(q \mid p)<i-\gamma$ and $K(b \mid q, a)<i-\gamma$. As before, this implies that there is no $q$ of complexity less than $2 n-3 i$ such that $K(q \mid p)<i-\gamma+O(\log n)$ and $K(b \mid q, a)<i-\gamma+O(\log n)$. Theorem 3 is proved.

Let us give one more example of a non-reducible description, more "geometric" than that of Theorem 3. Consider the two-dimensional vector space (the plane) over the finite field of cardinality $2^{n}$. The set $A$ now consists of all points of this plane and the set $B$ consists of all lines on it. The set $P$ consists also of all points of this plane. The function $\Xi$ is defined as follows: $\Xi(p, a)$ is the line passing through $a$ and $p$ (for $a \neq p$ ). This time $\alpha \approx 2^{n}$, as the line $a p_{1}$ coincides with the line $a p_{2}$ only if $a$ lies on the line $p_{1} p_{2}$ (in fact, small corrections are needed to take into account that $a \neq p_{1}$ and $a \neq p_{2}$ )

Let $Q=\{0,1\}^{1.5 n}$. For appropriate choice of $\beta, \varphi, \psi$ the conditions of Lemma 3 are satisfied. And the conditional complexity of $b=\Xi(a, p)$ given $a$ is at most $n+O(\log n)$, as there are about $2^{n}$ lines passing through any given point. Apply the winning strategy based on the function $\Xi$ against adversary's strategy from Section 2. The covered subset of $P \times A$ is small and can be enumerated given $n$. This implies that all the random pairs in $P \times A$ (those whose complexity is close to $4 n$ ) are not covered. Therefore we obtain the following result (taking into account that the line passing through a pair of random independent points is random):

any random line $b$ on the plane over the field of cardinality $2^{n}$ has conditional complexity $\approx n$ given every its random point $a$; every other random point $p$ on that line is a description of complexity $2 n$ for $b$ (given the point $a$ ) that cannot be reduced to a description of complexity $1.5 n$.

(More precisely, we should require that $a$ and $p$ are independent random points on $b$, i.e., $K(a, p \mid b) \approx 2 n$.)

The constructions of this section (except for being more intuitive) have the following advantage compared to proofs from Sections 2 and 3: The complexities $K(b \mid a)$ and $K(b \mid a, p)$ remain small even if we consider time-bounded version of Kolmogorov complexity, i.e., require that the running time of the machine finding the object from its description is bounded by a polynomial in $n$. And the non-reducible program exists even for complexity relativized by any oracle, as in Section 3. 


\section{References}

[1] Andrej A. Muchnik, Conditional complexity and codes, Theoretical Computer Science, 271 (2002), p. 97-109.

[2] M. Li and P.M.B. Vitányi, An Introduction to Kolmogorov Complexity and its Applications, Springer-Verlag, New York, 2nd Edition, 1997.

[3] A. Muchnik, A. Shen, N. Vereshchagin, M. Vyugin. Non-reducible descriptions for conditional Kolmogorov complexity. ECCC Report, TR04-054, Jun 29, 2004. See also: Theory and Applications of Models of Computation, Lecture Notes in Computer Science, Springer Berlin/Heidelberg, 3959 (2006), p. 308-317.

[4] M. Ustinov, Non-reducible description for conditional Kolmogorov complexity, In Russian. Problemy peredachi informatsii (Problems of information transmission), vol. 41, No. 3 (2005), p. 58-63. 